\title{
The Property of Continuity and Positively Definite Characteristic Function of Compound Poisson Distribution as The Sum of Geometric Distribution
}

\author{
Sherli Yurinanda', Ferra Yanuar ${ }^{1}$, Dodi Devianto1," \\ ${ }^{1}$ Department of Mathematics, Faculty of Mathematics and Natural Sciences, Andalas Univerrsity Padang 25163, West Sumatra Province, Indonesia \\ ${ }^{\star}$ Corresponding author: ddevianto@fmipa.unand.ac.id
}

\begin{abstract}
The compound Poisson distribution as the sum of independent and identically random variables from geometric distribution is characterized by using characteristic function. The characteristic function of this compound distribution is obtained by LaplaceStieltjes transform. It is provided a characterization of this compound distribution employing the properties of characteristic function as continuous and positively definite function.
\end{abstract}

Keywords

compound Poisson distribution, geometric distribution, characteristic function, uniformly continuous, positively definite function

\section{INTRODUCTION}

The compound Poisson distribution as the sum of geometric distribution is the sum of independent and identically random variables from geometric distribution with parameter $(p)$, where the number of these random variables has Poisson distribution with parameter $(\lambda)$. First, let us denote $X_{1}, X_{2}, \ldots, X_{N}$ be independent and identically random variables from geometric distribution and the sum of these random variables denoted as

$$
S=\sum_{i=1}^{N} X_{i}
$$

The random variable $S$ has compound Poisson distribution as the sum of geometric distribution with parameter $(\lambda, p)$. In addition, this sum of independent random variables can be defined as convolution of a distribution. Some theories of convolution have been developed by previous researcher on some articles, Devianto et al. (2015) have introduced convolution of generated random variable from exponential distribution with stabilizer constan. Another reasearch related to convolution is explained by Serfozo (1986) where the paper introduced compound Poisson approximation for sums of random variables. While Barbour et al. (1992) have explained compound Poisson approximation for sums of nonnegative random variables via Stein's method. The compound Poisson distribution has many application in particular on insurance risk models, where the property of governed model is derived by using characteristic function, one of the application has explained by Willmot and Lin (2001) that reviewed Lundberg approximations for compound distributions with insurance applications. While the theoretical of convolution and its property has mentioned by Devianto (2016) in the continuity properties of characteristic function from convolution of exponential distribution with stabilizer constant. The previous research results on property of convolution is to confirm that characteristic function has important rules on deriving the property of compound distribution.

In this paper, we study the characteristic function property from compound Poisson distribution as the sum of geometric distribution. In section 2, we will discuss about some properties of statistic measure from compound Poisson distribution as the sum of geometric distribution. While, in section 3 we will explain about continuity and positively definite characteristic function of compound Poisson distribution as the sum of geometric distribution employing its characterization.

\section{SOME PROPERTIES OF COMPOUND POIS- SON DISTRIBUTION AS THE SUM OF GEOMET- RIC DISTRIBUTION}

The random variabel $N$ has Poisson distribution with probability mass function

$$
P(N=n)=\frac{e^{-\lambda} \lambda^{n}}{n !}
$$

where $n=1,2,3, \ldots$ is the number of probability occurrence 


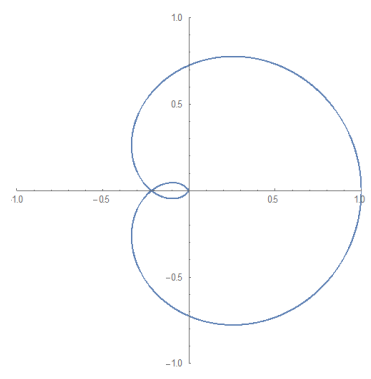

$\lambda=2$

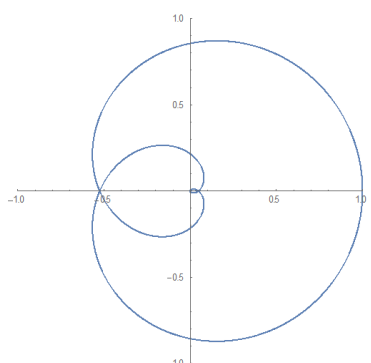

$\lambda=4$

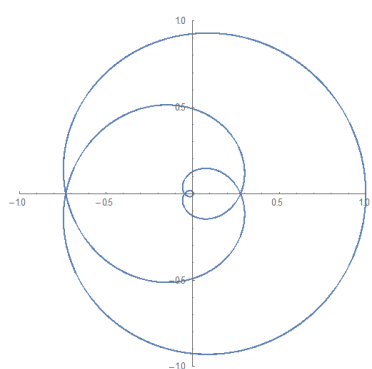

$\lambda=8$

Figure 1. Parametric curves of characteristic function from geometri distribution with various parameter $\lambda$

of events as $\mathrm{n}$ times with $\lambda$ is average of success event after many times trial. It has expectation, variance and moment generating function respectively as follows

$$
\begin{aligned}
& E(N)=\lambda \\
& \operatorname{Var}(N)=E(N-E(N))^{2}=\lambda \\
& M_{N}(t)=E(\exp (t N))=\exp \left(\lambda\left(e^{t}-1\right)\right)
\end{aligned}
$$

Another property of Poisson distribution is the characteristic function. The characteristic function is obtained by using Fourier-Stieltjes transform as explained in Lukacs (1992) or Sato (2013). The random variable $X$ has Poisson distribution with characteristic function

$$
\phi_{N}(t)=E(\exp (i t N))=\exp \left(\lambda\left(e^{i t}-1\right)\right)
$$

where and i as imaginary unit for $\exp (i t N)=\cos t N+i \sin t N$ and $i$ as imaginary unit for $t \in(-\infty, \infty)$.

We will explore the shape of parametric curves of characteristic function of Poisson distribution in Figure 1. The graphs of the characteristic function are described in the cartesian plane as a smooth line, this is to confirms graphically that its characteristic function is continuous.

The random variable $X$ has geometric distribution with parameter $\mathrm{p}$ defined as follows

$$
f(x)=p(1-p)^{x}
$$

for $x=1,2,3, \ldots$ The geometric distribution has expectation, variance, moment generating function and characteristic function respectively in the following term

$$
E(X)=\left(\frac{(1-p)}{p}\right)
$$

$$
\begin{aligned}
& V \operatorname{ar}(X)=E(X-E(X))^{2}=\frac{(1-p)}{p^{2}} \\
& M_{X}(t)=E(\exp (t X))=\frac{p}{1-(1-p) e^{t}} \\
& \phi_{X}(t)=E(\exp (i t X))=\frac{p}{1-(1-p) e^{i t}}
\end{aligned}
$$

The characterization of characteristic function from geometric distribution will explore from its shape of parametric curves in Figure 2. The following graphs are described in the cartesian plane that show a smooth line for each of parametric curves, this confirms graphically that the characteristic function from geometric distribution is continuous. The shape of parametric curves of characteristic function from geometric distribution tends to circle with radius of convergence tends to one when parameter $p$ tends to zero. Furthermore, the parametric curves of characteristic function from geometric distribution tends to a point at coordinate $(1,0)$ when the parameter $p$ tends to one.

The properties of compound Poisson distribution as the sum of geometric distribution in the terms of expectation, variance, moment generating function and characteristic function are given in the following proposition.

\section{Proposition 2.1.}

Let $S=\sum_{i=1}^{N} X_{i}$ be a random variable from compound Poisson distribution as the sum of geometric distribution, then expectation is obtained as follows

$$
E(S)=\frac{\lambda(1-p)}{p}
$$



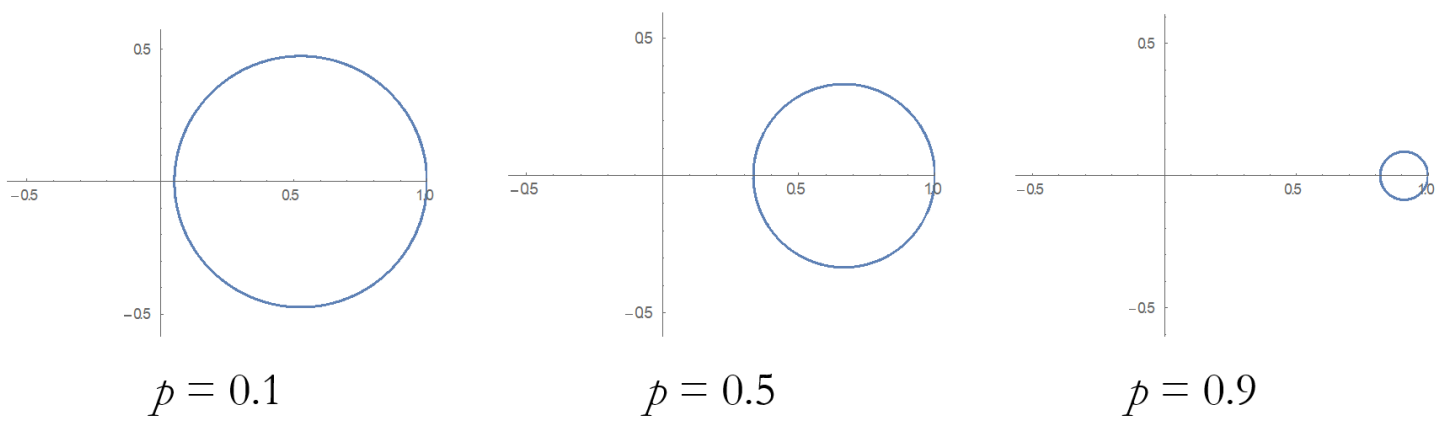

Figure 2. Parametric curves of characteristic function from geometric distribution with various parameters $p$

\section{Proof.}

By using definition of expectation and its linearity property then it is obtained

$$
\begin{aligned}
E(S) & =E\left(\sum_{i=1}^{N} X_{i}\right)=E\left(E\left(\sum_{i=1}^{N} X_{i} \mid N\right)\right) \\
& =E(N E(X))=E(N) E(X)
\end{aligned}
$$

It is used Equation (3) and (8), then we get

$$
E(S)=E(N) E(X)=\frac{\lambda(1-p)}{p}
$$

\section{Proposition 2.2}

Let $S=\sum_{i=1}^{N} X_{i}$ be a random variable from compound Poisson distribution as the sum of geometric distribution, then variance is obtained as follows

$$
\operatorname{Var}(S)=\frac{\lambda(1-p)(2-p)}{p^{2}}
$$

\section{Proof.}

By using definition of variance and linearity of expectation properties, then we get

$$
\begin{aligned}
\operatorname{Var}(S) & =E\left(\operatorname{Var}\left(\sum_{i=1}^{N} X_{i} \mid N\right)\right)+\operatorname{Var}\left(E\left(\sum_{i=1}^{N} X_{i} \mid N\right)\right) \\
& =E(N) \operatorname{Var}(X)+V \operatorname{ar}(N)(E(X))^{2}
\end{aligned}
$$

It is used Equation (3), (4), (8) and (9) then we have:

$$
\begin{aligned}
\operatorname{Var}(S) & =E(N) V \operatorname{ar}(X)+V \operatorname{ar}(N)(E(X))^{2} \\
& =\frac{\lambda(1-p)(2-p)}{p^{2}}
\end{aligned}
$$

\section{Proposition 2.3.}

Let $S=\sum_{i=1}^{N} X_{i}$ be a random variable from compound poisson distribution as the sum of geometric distribution, then moment generating function is obtained as follows

$$
M_{s}(t)=\exp \left(\frac{\lambda(1-p)\left(e^{t}-1\right)}{1-(1-p) e^{t}}\right)
$$

\section{Proof.}

By using definition of expectation and its linearity property then it is obtained for independent and identically random variable $X$ as follows

$$
\begin{aligned}
M_{S}(t) & =E\left(\exp \left(t \sum_{i=1}^{N} X_{i}\right)\right)=E\left(E\left(\exp \left(t \sum_{i=1}^{N} X_{i}\right) \mid N\right)\right) \\
& =E\left(M_{X}(t)^{N}\right)=M_{N}\left(\ln \left(M_{X}(t)\right)\right)
\end{aligned}
$$

It is used Equation (5) and (10) then we have

$$
\begin{aligned}
M_{s}(t) & =M_{N}\left(\ln \left(M_{X}(t)\right)\right) \\
& =M_{N}\left(\ln \left(\frac{p}{1-(1-p) e^{t}}\right)\right) \\
& =\exp \left(\frac{\lambda(1-p)\left(e^{t}-1\right)}{1-(1-p) e^{t}}\right)
\end{aligned}
$$




\section{Proposition 2.4 .}

Let $S=\sum_{i=1}^{N} X_{i}$ be a random variable from compound poisson distribution as the sum of geometric distribution, then characteristic function is obtained as follows

$$
\phi_{s}(t)=\exp \left(\frac{\lambda(1-p)\left(e^{i t}-1\right)}{1-(1-p) e^{i t}}\right)
$$

\section{Proof.}

By using definition of characteristic function and linearity of expectation then we have

$$
\begin{aligned}
\phi_{s}(t) & =E\left(\exp \left(i t \sum_{i=1}^{N} X_{i}\right)\right) \\
& =E\left(E\left(\exp \left(i t \sum_{i=1}^{N} X_{i}\right) \mid N\right)\right) \\
& =E\left(\phi_{X}(t)^{N}\right)=M_{N}\left(\ln \left(\phi_{X}(t)\right)\right)
\end{aligned}
$$

It is used Equation (5) and (9) then we have

$$
\begin{aligned}
\phi_{S}(t) & =M_{N}\left(\ln \left(\phi_{X}(t)\right)\right)=M_{N}\left(\ln \left(\frac{p}{1-(1-p) e^{i t}}\right)\right) \\
& =\exp \left(\frac{\lambda(1-p)\left(e^{i t}-1\right)}{1-(1-p) e^{i t}}\right)
\end{aligned}
$$

The parametric curve of characteristic function from compound Poisson distribution as the sum of geometric distribution will explore in Figure 3 and Figure 4 as follows. The characteristic function is continuous in the sense of graphically, since the graph of its characteristic function in the Cartesian plane is a smooth line.

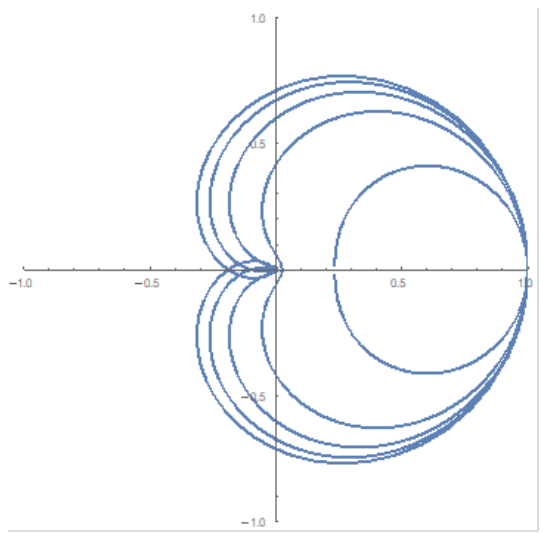

Figure 3. Parametric curves of characteristic function of $S$ with various parameters $p=0.1,0.3,0.5,0.7,0.9$ and $\lambda=8$

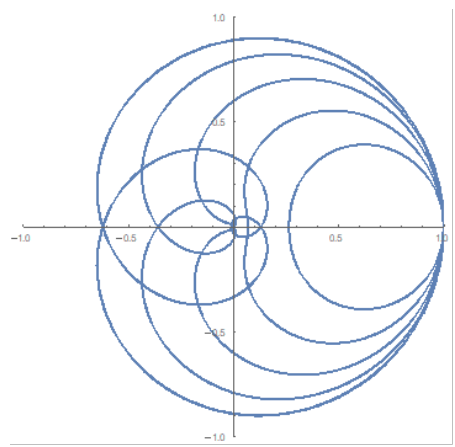

Figure 4. Parametric curves of characteristic function from $S$ with various parameters $\lambda=2,4,8,16,32$ and $p=0.5$

\section{THE PROPERTY OF CHARACTERISTIC FUNC- TION FROM COMPOUND POISSON DISTRIBU- TION AS THE SUM OF GEOMETRIC DISTRIBU- TION}

In this section we will state the main results to explain the uniform continuity and positively definite of characteristic function. The results are given in the following propositions.

\section{Proposition 3.1.}

Let $S=\sum_{i=1}^{N} X_{i}$ be a random variable from compound Poisson distribution as the sum of geometric distribution with parameter $(\lambda, p)$, then the characteristic function $\phi_{s}(t)$ is continuous.

\section{Proof.}

The continuity of characteristic function of compound Poisson distribution as the sum of geometric distribution is obtained by using definition of uniform continuity, that is for every $\varepsilon>0$ there exists $\delta>0$ such that $\left|\phi_{s}\left(t_{1}\right)-\phi_{s}\left(t_{2}\right)\right|<\varepsilon$ ) for $\left|t_{1}-t_{w}\right|<\delta$ where $\delta$ depends only on $\varepsilon$ Hence we have the following equation

$$
\begin{aligned}
\left|\phi_{S}\left(t_{1}\right)-\phi_{S}\left(t_{2}\right)\right| & =\mid \exp \left(\frac{\lambda(1-p)\left(e^{i t_{1}}-1\right)}{1-(1-p) e^{i t_{1}}}\right) \\
& -\exp \left(\frac{\lambda(1-p)\left(e^{i t_{2}}\right)}{1-(1-p) e^{i t_{2}}}\right) \mid
\end{aligned}
$$

Then let us define $h=t_{1}-t_{2}$, so that for $h \rightarrow 0$ we wave the following limit

$$
\begin{gathered}
\lim _{h \rightarrow 0} \mid \exp \left(\frac{\lambda(1-p)\left(e^{i\left(h+t_{2}\right)}-1\right)}{1-(1-p) e^{i\left(h+t_{2}\right)}}\right) \\
-\exp \left(\frac{\lambda(1-p)\left(e^{i t_{2}}-1\right)}{1-(1-p) e^{i t_{2}}}\right) \mid=0
\end{gathered}
$$

This hold for $\delta<\varepsilon$ where $\left|\phi_{S}\left(h+t_{2}\right)-\phi_{S}\left(t_{2}\right)\right|<\varepsilon$ for $\left|t_{1}-t_{2}\right|<$ $\delta$. Then $\phi_{S}(t)$ is the uniformly continuous. 


\section{Proposition 3.2}

Let $S=\sum_{i=1}^{N} X_{i}$ be a random variable from compound poisson distribution as the sum of geometric distribution with parameter $(\lambda, p)$, then characteristic function $\phi_{S}(t)$ is positively defined function with quadratic form

$$
\sum_{1 \leq j \leq n} \sum_{1 \leq l \leq n} c_{j} \overline{c_{l}} \phi_{S}\left(t_{j}-t_{l}\right) \geq 0
$$

for any complex number $c_{1}, c_{2}, \ldots, c_{n}$ and real number $t_{1}, t_{2}, \ldots, t_{n}$.

\section{Proof}

It will show that charcteristic function $\phi_{S}$ is positive function in the quadratic form $\sum_{1 \leq j \leq n} \sum_{1 \leq l \leq n} c_{j} \overline{c_{l}} \phi_{X}\left(t_{j}-t_{l}\right) \geq 0$. Next, we have the following equation

$$
\begin{array}{r}
\sum_{1 \leq j \leq n} \sum_{1 \leq l \leq n} c_{j} \overline{c_{l}} \phi_{S}\left(t_{j}-t_{l}\right)= \\
\sum_{1 \leq j \leq n} \sum_{1 \leq l \leq n} c_{j} \overline{c_{l}}\left(\exp \left(\frac{\lambda(1-p)\left(e^{i\left(t_{j}-t_{l}\right)}-1\right)}{1-(1-p) e^{i i^{i\left(t_{j}-t_{l}\right)}}}\right)\right)= \\
\sum_{1 \leq j \leq n} \sum_{1 \leq l \leq n} c_{j} \overline{c_{l}}\left(\exp \left(\lambda\left(e^{i\left(t_{j}-t_{l}\right)}-1\right) \frac{(1-p)}{1-(1-p) e^{i\left(t_{j}-t_{l}\right)}}\right)\right)
\end{array}
$$

By using inequality of exponential properties and two constrains bellow

$$
\begin{aligned}
& \left(\lambda\left(e^{i\left(t_{j}-t_{l}\right)}-1\right)\right)>1 \\
& \left(\frac{(1-p)}{1-(1-p) e^{i i^{i\left(t_{j}-l_{l}\right)}}}\right)>1
\end{aligned}
$$

Then we can write equation 27 in the following inequality form

$$
\begin{array}{r}
\sum_{1 \leq j \leq n} \sum_{1 \leq l \leq n} c_{j} \overline{c_{l}} \phi_{S}\left(t_{j}-t_{l}\right) \geq \\
\sum_{1 \leq j \leq n} \sum_{1 \leq l \leq n} c_{j} \overline{c_{l}}\left(\exp \left(\lambda\left(e^{i\left(t_{j}-t_{l}\right)}-1\right)\right)\right) \\
+\sum_{1 \leq j \leq n} \sum_{1 \leq l \leq n} c_{j} \overline{c_{l}}\left(\exp \left(\frac{(1-p)}{1-(1-p) e^{i i^{i\left(t_{j}-t_{l}\right)}}}\right)\right)
\end{array}
$$

Next, the right side of the inequality (Equation 30 ) will be proven greater than zero for both of part in summation. Let us define the both part in the following notations

$$
S P_{1}=\sum_{1 \leq j \leq n} \sum_{1 \leq l \leq n} c_{j} \overline{c_{l}}\left(\exp \left(\lambda\left(e^{i\left(t_{j}-t_{l}\right)}-1\right)\right)\right)
$$

and

$$
S P_{2}=\sum_{1 \leq j \leq n} \sum_{1 \leq l \leq n} c_{j} \overline{c_{l}}\left(\exp \left(\frac{1-p}{1-(1-p) e^{i^{i\left(t_{j}-t_{l}\right)}}}\right)\right)
$$

From equation 31 , it is used definition of characteristic function and the property of expectation then we obtained that

$$
\begin{aligned}
S P_{1} & =\sum_{1 \leq j \leq n} \sum_{1 \leq l \leq n} c_{j} \overline{c_{l}}\left(\exp \left(\lambda\left(e^{i\left(t_{j}-t_{l}\right)}-1\right)\right)\right) \\
& =\sum_{1 \leq j \leq n} \sum_{1 \leq l \leq n} c_{j} \overline{c_{l}} E\left(\exp \left(i\left(t_{j}-t_{l}\right) X\right)\right. \\
& =\left(\sum_{1 \leq j \leq n} c j E\left(\exp \left(i t_{j} X\right)\right)\right)\left(\sum_{1 \leq l \leq n} \overline{c_{l}} E\left(\exp \left(-i t_{l} X\right)\right)\right)
\end{aligned}
$$

Furthermore we use the definition of complex conjugate on characteristic function and its modulus property, then we can rewrite the equation 33 in the following form

$$
\begin{aligned}
S P_{1} & =\left(\sum_{1 \leq j \leq n} c j E\left(\exp \left(i t_{j} X\right)\right)\right)\left(\overline{\sum_{1 \leq l \leq n} c_{l} E\left(\exp \left(i t_{l} X\right)\right)}\right) \\
& =\left|\sum_{1 \leq j \leq n} c j E\left(\exp \left(i t_{j} X\right)\right)\right|^{2}
\end{aligned}
$$

Next, from equation (32), it use definition of characteristic function and some algebraic manipulation, then we obtained that

$$
\begin{aligned}
S P_{2} & =\sum_{1 \leq j \leq n} \sum_{1 \leq l \leq n} c_{j} \overline{c_{l}}\left(\exp \left(\frac{1-p}{1-(1-p) e^{i i^{i\left(t_{j}-t_{l}\right)}}}\right)\right) \\
& =\sum_{1 \leq j \leq n} \sum_{1 \leq l \leq n} c_{j} \overline{c_{l}}\left(\exp \left(\frac{1-p}{p} \frac{p}{1-(1-p) e^{i^{i\left(l_{j}-t_{l}\right)}}}\right)\right) \\
& \geq \sum_{1 \leq j \leq n} \sum_{1 \leq l \leq n} c_{j} \overline{c_{l}} \exp \left(\frac{1-p}{p}\right) \\
& +\sum_{1 \leq j \leq n} \sum_{1 \leq l \leq n} c_{j} \overline{c_{l}}\left(\exp \left(\frac{p}{1-(1-p) e^{i i^{i\left(t_{j}-t_{l}\right)}}}\right)\right)
\end{aligned}
$$

Furthermore we use the definition arthmatics series and property of complex conjugate in characteristic function on equation 
35 then we have

$$
\begin{aligned}
S P_{2} & \geq n \exp \left(\frac{1-p}{p}\right)+\sum_{1 \leq j \leq n} \sum_{1 \leq l \leq n} c_{j} \overline{c_{l}} E\left(\exp \left(i\left(t_{j}-t_{l}\right) X\right)\right. \\
& =n \exp \left(\frac{1-p}{p}\right)+\left(\sum_{1 \leq j \leq n} c j E\left(\exp \left(i t_{j} X\right)\right)\right) \\
& \left(\overline{\sum_{1 \leq l \leq n} c_{l} E\left(\exp \left(i t_{l} X\right)\right)}\right) \\
& =n \exp \left(\frac{1-p}{p}\right)+\left|\sum_{1 \leq j \leq n} c j E\left(\exp \left(i t_{j} X\right)\right)\right|^{2}
\end{aligned}
$$

From Inequality (equation 33 and 34) we obtain that

$$
\begin{array}{r}
\sum_{1 \leq j, l \leq n} \sum_{1 \leq l \leq n} c_{j} \overline{c_{l}} \phi_{s}\left(t_{j}-t_{l}\right) \geq\left|\sum_{1 \leq j \leq n} c j E\left(\exp \left(i t_{j} X\right)\right)\right|^{2} \\
+n \exp \left(\frac{1-p}{p}\right)+\left|\sum_{1 \leq j \leq n} c j E\left(\exp \left(i t_{j} X\right)\right)\right|^{2}
\end{array}
$$

for every

$$
n \exp \left(\frac{1-p}{p}\right) \geq 0 \text { and } n=1,2,3, \ldots
$$

So we can conclude that $\sum_{1 \leq j \leq n} \sum_{1 \leq l \leq n} c_{j} \overline{c_{l}} \phi_{s}\left(t_{j}-t_{l}\right) \geq 0$. Then it proved that $\phi_{s}(t)$ as positively defined function where the quadratic form has nonnegative values.

\section{CONGLUSION}

We can conclude that characteristic function of compound poisson distribution as the sum of geometric distribution is obtained as $\phi_{s}(t)$ with parameter $(\lambda, p)$ This characteristic function is continuous and positively defined function as explained in Proposition 3.1 and Proposition 3.2.

\section{REFERENCES}

Barbour, A. D., L. H. Y. Chen, and W.-L. Loh (1992). Compound Poisson Approximation for Nonnegative Random Variables Via Stein's Method. The Annals of Probability, 20(4); 1843-1866

Devianto, D. (2016). The uniform continuity of characteristic function from convoluted exponential distribution with stabilizer constant. In AIP Conference Proceedings, volume 1707. AIP Publishing LLC, page 080006

Devianto, D., Maiyastri, L. Oktasari, and M. Anas (2015). Convolution of generated random variable from exponential distribution with stabilizer constant. Applied Mathematical Sciences, 9; 4781-4789

Lukacs, E. (1992). Characteristic Function. Hafner Publishing Company

Sato, K. (2013). Levy Processes and Infinitely Divisible Distributions. New York: Cambridge Studies in Advance Mathematics

Serfozo, R. F. (1986). Compound Poisson Approximations for Sums of Random Variables. The Annals of Probability, 14(4); 1391-1398

Willmot, G. E. and X. S. Lin (2001). Lundberg Approximations for Compound Distributions with Insurance Applications. Springer New York 